\title{
Wittgenstein y la justificación de las proposiciones gozne*
}

\section{Wittgenstein and the justification of hinge propositions}

\author{
Modesto Gómez-Alonso \\ G.I. Epistemology of Religious Belief: \\ Wittgenstein, Grammar and the Contemporary World \\ Facultad de Filosofía \\ Universidad Pontificia de Salamanca \\ Salamanca, España \\ EIDYN Research Centre \\ University of Edinburgh. \\ Edinburgh, UK \\ E-mail: mgomezal@upsa.es \\ ORCID: 0000-0001-6889-2330
}

Fecha de recepción: 12 de septiembre de 2018 Fecha de aprobación: 29 de octubre de 2018

Doi: $10.17533 / 10.17533 /$ udea.ef.n60a08

Resumen. Las cuestiones acerca de la posibilidad del pensamiento, entendido como una actividad dirigida a la realidad, son más profundas que las referidas a si el conocimiento es posible. El trilema de Agripa, particularmente mediante el tropo de arbitrariedad, expresa dichas cuestiones intuitivamente. Este tropo es, además, operativo en lo que se refiere a la epistemología de goznes, principalmente en virtud del hecho de que los goznes wittgensteinianos son principios fundacionales de nuestra práctica epistémica. Como tales, carecen necesariamente de aval racional, lo que plantea el problema de si la arbitrariedad de su aseveración es inherente a su carácter fundacional. Para afrontar este reto, los epistemólogos de goznes han propuesto una serie de argumentos prácticos que conciben los goznes bien como postulados que se exigen legitimamente a cualquier agente epistémico o como reglas constitutivas de la práctica ordinaria. El objeto de este trabajo es el de demostrar que tales argumentos son presa de consideraciones escépticas.

Palabras clave: constitutivismo, epistemología de goznes, escepticismo semántico, justificación racional, presuposición arbitraria, primacía de la práctica, Wittgenstein

* Este artículo forma parte del Proyecto de Investigación "Epistemology of Religious Belief: Wittgenstein, Grammar and the Contemporary World" (PTDC/FER-FIL/32203/2017), financiado por la Portuguese Foundation for Science and Technology (FCT).

Cómo citar este artículo:

MLA: Gómez-Alonso, Modesto. "Wittgenstein y la justificación de las proposiciones gozne". Estudios de Filosofia 60 (2019): 159-182.

APA: Gómez-Alonso, M. (2019). Wittgenstein y la justificación de las proposiciones gozne. Estudios de Filosofia, 60, pp. 159-182.

Chicago: Modesto Gómez-Alonso. "Wittgenstein y la justificación de las proposiciones gozne". Estudios de Filosofía n. ${ }^{\circ} 60$ (2019): $159-182$. 
Abstract. Questions about the possibility of thought as an activity directed to reality, are deeper than those concerning the possibility of knowledge. These deeper questions have found intuitive expression in the Agrippan Trilemma, particularly in the trope of arbitrary presupposition. This trope can be raised as a criticism of current varieties of hinge epistemology inspired by Wittgenstein, mainly by virtue of the fact that Wittgensteinian hinges are foundational principles governing our epistemic practice. As such, they are necessarily unsupportable, being thus difficult to see how it would be possible to conciliate the hinges' lack of support with their being not bare, arbitrary assertions. Facing up to this challenge, hinge epistemologists have proposed practical - though not pragmatic - arguments according to which hinges are either postulates legitimately demanded to every epistemic agent or rules constitutive of quotidian practices. It is the aim of this article to show that practical arguments for hinges fall short, necessarily so.

Keywords: arbitrary assumption, constitutivism, hinge epistemology, meaning-scepticism, primacy of the practical, rational justification, Wittgenstein

Mi respeto por los talentos de Maimon no conoce límites. Creo firmemente que ha demolido al completo la filosofía de Kant tal como todo el mundo, usted incluido, la habia entendido hasta ahora -y estoy preparado para demostrarlo. Nadie ha tomado en consideración lo que ha hecho. Todos lo han despreciado desde sus alturas. Pienso que por eso mismo los siglos futuros se burlarán amargamente de nosotros.

Carta de Fichte a Reinhold, marzo/abril 1795 (Fichte, 1988, pp. 383-4)

\section{Presuposiciones wittgensteinianas}

En un estudio ya clásico acerca de los tropos pirrónicos y de las herramientas lógicas a disposición del escepticismo, Jonathan Barnes (Barnes, 1990) distingue dos significados del término presuposición, $\mathrm{y}$, en consecuencia, dos tradiciones acerca de la función filosófica que cumple 'asumir', 'presuponer' o 'dar por supuesta' la verdad de una proposición P.

De acuerdo con la tradición platónica, la función de una presuposición es fundamentalmente heurística, o, en otras palabras, su cometido es el de — dentro del marco de una investigación específica - adelantar o dar provisionalmente por supuesta (sin argumentación) una respuesta y analizar sus consecuencias. Por ejemplo, la cuestión que se investiga en el Teeteto es la de qué es el conocimiento, y lo que en un determinado momento del diálogo se asume es que el conocimiento es juicio verdadero (Platón, 2014). Posteriormente, una serie de contraejemplos muestran que esta definición es inadecuada. Lo importante aquí es que la proposición que se presupone cumple la función de una mera hipótesis, que o bien está a la espera de justificación o, en el marco de una reducción al absurdo, de ser invalidada. Lo que significa, por una parte, que el investigador únicamente da por supuesta la 
verdad de $\mathrm{P}$ pro tempore y en el contexto de la argumentación - sin comprometerse con su verdad y sin aseverarla en un sentido estricto-, y, por otra, que dicha presuposición, lejos de tratarse de la base y del punto de partida sin los cuales ni la argumentación ni la búsqueda de verdades serían posibles, es un instrumento intermedio en el proceso de búsqueda de verdades particulares. Es obvio además que, de acuerdo con la tradición platónica, qué proposiciones puedan presuponerse razonablemente dependerá de factores contextuales que incluyen el objeto de investigación y la posición epistémica del investigador.

Por su parte, la tradición aristotélica entiende la presuposición en un sentido muy diferente — no en referencia a una proposición que se adelanta sin argumentos con el objeto de, mediante el examen de sus consecuencias, ser o no abandonada, sino como actitud de asentimiento respecto a proposiciones que, básicas, fundacionales y racionalmente primitivas, desempeñan la función de principios lógicos o epistémicos. La presuposición no es, por tanto, temporal, sino necesaria. El principio no está a la espera de razones a favor o en contra, sino que se encuentra situado por definición más allá del espacio argumentativo. Lo que damos por supuesto no es una teoría particular que actúa como medio o vehículo de descubrimiento, sino un elemento incluido entre las condiciones de posibilidad mismas del juicio y del proceso argumentativo. De lo que se sigue, por una parte, que las presuposiciones aristotélicas implican el compromiso del sujeto con la verdad de la proposición - tratándose además de un compromiso exigido por el compromiso con la racionalidad misma-, y, por otra, que son independientes de factores circunstanciales y contingentes tales como la posición epistémica particular del sujeto o el objeto concreto de investigación. Podríamos decir, en consecuencia, que contamos con dos criterios para distinguir los posibles objetos de una presuposición aristotélica. En primer lugar, un criterio puramente lógico o formal - y negativo - de acuerdo al cual ninguna proposición de la que pueda darse razones (a favor o en contra) es objeto de presuposición aristotélica. En segundo lugar, un criterio más sustantivo que señala que el objeto de presuposición debe ser constitutivo de la racionalidad. Es este último aspecto el que, fundamentalmente, consideraremos.

Pese a las numerosas controversias acerca de la extensión y naturaleza de las llamadas 'proposiciones-gozne' del Sobre la certeza de Wittgenstein, pienso que su caracterización se adecua fundamentalmente a la de las presuposiciones aristotélicas. Por lo pronto, Wittgenstein señala explícitamente que, lejos de tratarse de hipótesis (Wittgenstein, 2004, § 402) o de resultados alcanzados a partir de una 
investigación (Wittgenstein, 2004, §138), dichas 'proposiciones' conforman "el punto de partida de la creencia" (Wittgenstein, 2004, § 210), y cumplen una "función lógica" (Wittgenstein, 2004, § 136) tal que, en lo que a ellas respecta, la ausencia de duda constituye la condición de posibilidad del juicio (Wittgenstein, 2004, $\S \S$ 308, 419, 670). Wittgenstein también deja claro que dichos principios configuran una categoría epistémica invariable, y que, en consecuencia, son independientes de la situación cognitiva del sujeto — criticando a Moore precisamente por el hecho de haber transformado en principios epistémicos certezas empíricas cuya indubitabilidad depende de factores circunstanciales (Wittgenstein, 2004, § 155), y por haber cometido así un error categorial consistente en la confusión entre "los fundamentos de todas nuestras operaciones con el pensamiento (y con el lenguaje)" (Wittgenstein, 2004, § 401) y juicios particulares. Añádase que se trata de presuposiciones sin fundamento - más allá del espacio de razones - cuya verdad debemos asumir o dar por supuesta (Wittgenstein, 2004, $\S \S 210,253$ ), y que Wittgenstein desarrolla el criterio de distinción formal mencionado arriba como criterio de demarcación entre goznes y proposiciones empíricas (Wittgenstein, $2004, \S 307)$, para que la analogía sea completa.

Lo que, sin embargo, me interesa subrayar ahora son dos aspectos de los goznes wittgensteinianos. En primer lugar, el que se trata de proposiciones que, aunque cumplen una función lógica, poseen la forma de proposiciones empíricas (Wittgenstein, 2004, § 401). O, lo que es lo mismo, el hecho de que son proposiciones que, a diferencia de principios meramente formales del pensamiento como el principio de contradicción, poseen un contenido semántico sustantivo. Tres ejemplos especialmente relevantes de goznes son, en este sentido, la existencia del mundo externo (Wittgenstein, 2004, $\S \S 20,476$ ), la existencia de un orden o secuencia temporal objetivos (Wittgenstein, 2004, $\S \S 183-92$ ) y el principio general de causalidad como marco de inteligibilidad de la experiencia (Wittgenstein, 2004, $\S 613$ ). Como puede comprobarse, esto supone una extensión (problemática) de las nociones aristotélicas de 'lógica' y de 'principio', extensión que, además, parece poseer una marcada dirección trascendental (de acuerdo con la distinción kantiana entre lógica formal y lógica trascendental).

En segundo lugar, Wittgenstein observa, frente a la prueba del mundo externo de Moore, que ninguna creencia empírica (incluidas proposiciones a las que otorgamos nuestro asentimiento en circunstancias cognitivas óptimas, por ejemplo "Tengo dos manos") puede servir para justificar la verdad de un gozne como la existencia del mundo externo (Wittgenstein, 2004, § 210). Esto se debe, 
por una parte, a que sólo asumiendo la verdad de los goznes podemos otorgarles peso y direccionalidad epistémicas a las experiencias sobre las que dichas creencias (empíricas) se basan, de forma que una experiencia subjetiva pueda — dadas esas presuposiciones - transformarse en razón o evidencia a favor de P. En otras palabras: una experiencia tal como si tuviese manos contaría racionalmente como indicador de la verdad de mi creencia de que tengo manos únicamente en la medida en que presupongo, entre otras cosas, que existe un mundo externo. Por otra parte, y como ya hemos señalado, el propio carácter fundacional y primitivo de los goznes conlleva un orden asimétrico por el que los principios de la racionalidad epistémica — para ser principios - no pueden ser justificados por aquello a lo que hacen posible. Dicha justificación revertiría el orden epistémico y cancelaría los conceptos mismos de 'fundación', 'gozne' y 'presuposición'.

El problema radica, sin embargo, en la propia definición de los goznes como presuposiciones injustificables. La epistemología antigua resulta, también en este punto, ilustrativa. Uno de los tropos de Agripa — el tropo de arbitrariedad — ataca directamente las presuposiciones aristotélicas, y lo hace precisamente porque, al no poder estar basadas en razones, se trataría de meras aseveraciones carentes de estatus epistémico, tan legítimas (o tan poco legítimas) como la presuposición de proposiciones contrarias cuya verdad excluya la verdad de dichos principios. Este conflicto entre presuposiciones igualmente arbitrarias desembocaría en un caso límite de isostheneia o equilibrio epistémico, en el que las dos aseveraciones opuestas - y que, sin embargo, cuentan (ambas) formalmente como candidatos legítimos a principios básicos - estarían a la par, no en lo que respecta al peso de las razones que las apoyan, sino a la ausencia, en ambos casos, de razones de tipo alguno. Como es bien sabido, la actitud racional que la isostheneia exige es la suspensión del juicio.

Es importante subrayar, en relación con lo anterior, que la función de los escenarios construidos por el escepticismo moderno y contemporáneo (desde el Dios engañador al cerebro en la probeta) no es la de proporcionar razones a favor de una hipótesis negativa (que nuestras creencias son masivamente falsas), sino la de hacer comprensible de forma intuitiva cómo la totalidad de nuestra experiencia es compatible con la falsedad de las creencias fundamentales, $\mathrm{y}$, por tanto, la de hacer visible la arbitrariedad de tales compromisos básicos. En cualquier caso, la presión del escéptico obliga al dogmático - sea aristotélico, estoico o wittgensteiniano-a buscar una vía media entre la aseveración arbitraria y una justificación que invierta el orden epistémico y trate de legitimar principios con base a evidencias que sólo 
cuentan como evidencias en la medida en que se presupone la verdad de esos principios. En caso contrario, se cierra la red escéptica.

Lo que, pese a sus diferencias internas, unifica las propuestas de Crispin Wright (2004), Duncan Pritchard (2016) y Annalisa Coliva (2015), y permite clasificarlas bajo el rótulo común de epistemología de goznes (Hinge Epistemology), es, además de la inspiración común en Wittgenstein, la primacía que otorgan a la dimensión práctica de la racionalidad epistémica en sus respuestas al escepticismo y en la instauración de la vía media a la que nos hemos referido. Podría hablarse incluso de un giro práctico en epistemología, siempre que no se confunda esta expresión con un supuesto 'giro pragmático' o con una forma de argumentación 'consecuencialista', y que se la entienda dentro de un horizonte eminentemente kantiano.

El punto de partida es una concesión — a veces elaborada y explícita (Wright, 1991, p. 89), otras reticente y disimulada (Coliva, 2015, p. 145) - al escepticismo: lo que se constata es que desde el punto de vista de la pura racionalidad teórica, y en la medida en que los escenarios escépticos son siempre metafisicamente posibles, no estamos racionalmente obligados a comprometernos con la verdad de los goznes. Sin embargo, la demanda a actuar y formar racionalmente nuestras creencias podrá satisfacerse sólo si se supone la eficacia y causalidad de dicha demanda, o, en otras palabras, el compromiso con el mandato epistémico a actuar racionalmente lleva implícito el compromiso con las condiciones de realización de ese mandato, que, por supuesto, no son otras que los goznes — para que haya deber racional éste ha de poder cumplirse: deber implica poder. En consecuencia, la afirmación de los goznes es algo que se requiere y espera de todo agente racional por el mero hecho de serlo (Wittgenstein, 2004, § 254). Y eso aunque una duda sobre los goznes siempre sea teóricamente posible (Wittgenstein, 2004, § 392), y, por ello, aunque no nos encontremos racionalmente obligados a su afirmación.

Respecto al argumento precedente, nótese, en primer lugar, que la norma o mandato epistémico es, en terminología kantiana, además de práctico, puro. Lo que significa que es un mandato cuya fuente es la racionalidad, y, por tanto, que la motivación para afirmar la realidad de los goznes no es, tal como dijimos arriba, pragmática, sino constitutiva de la agencia racional misma. Nótese, además, y en virtud de lo anterior, que la crítica al escepticismo no consiste en una nueva versión del cargo de apraxia en la que lo que se aduce es que éste conduce a la parálisis 
cognitiva y ha de ser rechazado por sus consecuencias nihilistas, sino en hacernos ver que al dudar de los goznes lo que el escéptico pone en duda es la posibilidad de actuar racionalmente, $y$, por tanto, el propio deber racional de hacerlo. Como el escéptico apela a su deber racional para justificar la suspensión del juicio, su ataque a los goznes revierte sobre él mismo, implosionando su posición.

Por otra parte, Annalisa Coliva (2015, pp. 128-9) elabora el tema de la primacía de la práctica mediante un procedimiento inverso, cuyo punto de partida son nuestras prácticas epistémicas diarias. Su argumentación consiste en una deducción trascendental en la que a partir de los hechos (i) de que los compromisosgozne son inherentes a nuestras prácticas epistémicas cotidianas de buscar y sopesar evidencias, proporcionar razones para nuestras creencias empíricas, justificar y poner en duda opiniones, etc., y (ii) de que el escéptico admite — en un sentido, cuanto menos, 'estrecho' - la racionalidad de esas prácticas; se establece una noción extensa de racionalidad que incluye en su naturaleza sus condiciones de posibilidad, racionalidad extensa que, a su vez, fuerza al escéptico a aceptar los goznes como consecuencia de su aceptación de la racionalidad de las prácticas epistémicas ordinarias.

Me resulta, sin embargo, curiosa la aparente circularidad de este argumento, que cita la direccionalidad epistémica de la experiencia como razón a favor de la verdad de los goznes, verdad que, a su vez, es la razón que justifica que la experiencia posea direccionalidad epistémica; y que apela a la práctica para justificar la práctica. Por eso pienso que no se trata de un argumento justificativo - lo que supondría tanto la reversión del orden epistémico como una petición de principio-, sino de un argumento explicativo que considera un determinado fenómeno como dado, y cuya función es la de descartar cualquier explicación de nuestras prácticas epistémicas que implique el escepticismo sobre las mismas. El objeto de crítica es, por tanto, Hume, y con él, las explicaciones subjetivistas, fenomenistas y deflacionarias de la normatividad de nuestros juegos de lenguaje cognitivos.

Hemos señalado ya la impronta kantiana que en la epistemología de goznes adquiere Wittgenstein. Este aspecto se refuerza - a mi entender correctamenteen la versión de Coliva, quien se compromete explícitamente con una concepción no-realista de la verdad (Coliva, 2015, p. 37), y, por ello, reubica la problemática escéptica dentro de un marco trascendental donde lo que está en cuestión no es si los goznes se corresponden a un mundo-en-sí, sino su objetividad trascendental 
-que se trate de elementos objetivos y constitutivos del trasfondo epistémico y agencial, y no de la expresión subjetiva de nuestros deseos- y su capacidad para constituir objetos de conocimiento y para servir de reglas que guíen nuestras prácticas cotidianas. En otras palabras, de lo que se trata es de examinar los goznes en tanto que principios epistémicos, y no ontológicos.

Por eso es importante subrayar que, más que interpretar la posibilidad de escenarios escépticos de forma realista, debemos circunscribirlos al ámbito de la racionalidad trascendental, como indicadores intuitivos de la posible falsedad trascendental de los goznes. Su posibilidad metafísica es, por tanto, la posibilidad de que la experiencia no sea, en sentido kantiano, objetiva. También por eso es importante distinguir entre dos formas de considerar trascendentalmente los goznes: bien como postulados de la razón epistémica, o como categorías o reglas que dirigen y constituyen las prácticas cognitivas empíricas. Un argumento justificativo cuyo objeto sea su legitimación racional los considerará en el primer sentido. Un argumento explicativo — análogo al de Coliva — que se limite a explicitar aspectos inherentes a la práctica que la hacen posible y a mostrar la conexión interna de reglas y práctica, en el segundo.

Lo que trataré de mostrar a lo largo de este trabajo es que ambas argumentaciones son inválidas, y que, incluso dando por supuesta la reinterpretación kantiana sobre la que acabamos de llamar la atención, la teoría de Wittgenstein cae presa de consideraciones escépticas. Nuestro propósito es, en consecuencia, la elaboración de una crítica inmanente a la epistemología de goznes.

En la siguiente sección se mostrará cómo la ausencia de un criterio que permita diferenciar postulados prácticos y consideraciones pragmáticas (y wishful thinking) impide la validación racional de los goznes y la adquisición del estatus epistémico objetivo que ésta implicaría. En la sección 3, plantearemos las cuestiones Quid Juris? (¿con qué derecho?) y Quid facti? (¿qué hecho?), no en lo que se refiere a la objetividad trascendental de los goznes, sino, la primera, a la transparencia e inteligibilidad de su aplicación categorial a la experiencia, y la segunda, a su realidad objetiva (y no meramente simbólica), es decir, a si se trata de reglas reales de uso, capaces de determinar actitudes empíricas específicas y de servir de fundamento de juicios particulares. Aquí daremos por supuesto que los goznes son formas de pensamiento que, aunque racional o trascendentalmente problemáticas, son necesarias para una constitución mínima de la experiencia, pero nos plantearemos 
si y cómo principios tan generales y abstractos pueden conferir orden objetivo o peso evidencial a los fenómenos, $\mathrm{y}$, por tanto, explicar la normatividad epistémica y la determinación de juicios in concreto. Se trata, por tanto, del problema de la transición de lo trascendental a lo empírico. Un problema común a cualquier forma de deducción trascendental de categorías, sea kantiana o wittgensteiniana.

Lo que, como poco, las respuestas a Quid Juris? y Quid facti? sugieren es que, porque no es evidente que el significado empírico de las creencias fundamentales se corresponda con su significado trascendental, y porque su origen puede deberse a una regularidad de facto e inestable (y no de iure) en el cumplimiento de nuestras expectativas epistémicas, una explicación escéptica de nuestras prácticas es, contra Coliva, perfectamente posible. Lo que significaría, tal vez, que los goznes (al menos, en su empleo ordinario y en lo que respecta a su dependencia de lo que contingentemente suceda) sean más similares a hipótesis platónicas que a presuposiciones aristotélicas.

Curiosamente, y pese al marco trascendental en el que desarrolla su investigación, Wittgenstein parece haber sido consciente de las dificultades inherentes a las preguntas Quid Juris? y Quid facti? Como lo demuestran sus reflexiones - y fluctuaciones - acerca de la posibilidad de una 'pesadilla metafísica' consistente en el trastorno general de la naturaleza (Wittgenstein, 2004, §§ 513, 517). Sea como fuere, la fantasmagoría arbitraria y subjetiva, la ausencia completa de un orden a priori de las representaciones, que tanto temió Kant, reaparecen en el modelo de Wittgenstein. La primacía de la práctica no ofrece, en ninguno de sus sentidos, una vía media transitable.

Me resta señalar, para finalizar este apartado, que, al igual que sucede en el pirronismo, las conclusiones escépticas que alcancemos serán provisionales y contextuales, circunscritas únicamente a los argumentos dogmáticos bajo examen. No permiten descartar, por tanto, la posibilidad de una validación racional de los goznes. Aunque sí permiten concluir que, de ser posible, se trataría de una forma de validación muy diferente, y posiblemente mucho más exigente, a la que proponen los filósofos post-wittgensteinianos. En este sentido, concibo el escepticismo, más que como amenaza, como impulso que obliga a abrir nuevas vías filosóficas y que impide el estancamiento dogmático. Con sus dudas, el escéptico es un benefactor de la humanidad. 


\title{
2. La primacía de la práctica y los postulados de la racionalidad epistémica
}

\author{
Respondiendo a sus críticos, Crispin Wright escribe:
}

Si la presuposición de que existe un mundo material externo fuese realmente constitutiva de nuestra concepción de una investigación empírica racional, entonces debería existir algún tipo de ininteligibilidad en el cuestionamiento escéptico de la racionalidad de dicho presupuesto, ininteligibilidad que contradiría el sentido de paradoja que los mejores argumentos escépticos generan (Wright, 2012, p. 479; la traducción es del autor).

El texto de Wright apunta a algo que ya hemos señalado: la opacidad racional de los goznes wittgensteinianos, el hecho de que desde un punto de vista puramente teórico no sean proposiciones a cuya afirmación estemos racionalmente obligados. Pero también apunta, tal como la elección del término 'paradoja' indica en referencia a cómo vivimos las conclusiones escépticas, a un conflicto entre la inteligibilidad escéptica y algo más. Este 'algo más' es la práctica epistémica, o, mejor dicho, la práctica epistémica en tanto que se dirige a y culmina en la actividad de juzgar; donde el juicio es un acto de determinación racional de la voluntad —en una dirección bien de asentimiento o de negación- cuyo objeto no es tan solo dar en el blanco de la verdad, sino hacerlo de forma reflexiva, racional y responsable (Sosa, 2015, pp. 67-8), y que entraña un compromiso basado en razones con lo que declaramos y su verdad.

La paradoja consiste así en la combinación de la inteligibilidad del escepticismo con el hecho de que esta posición cancela la posibilidad de juicio (y de compromiso racional), y, por ello, la posibilidad tanto de adquisición de bienes epistémicos como de una transición entre voluntad racional y naturaleza, normatividad e impulso, libertad y determinación, noúmeno y fenómeno, que - supuestamente- sólo la posibilidad de juicio legitima. Lo que el escepticismo anula es la producción de una apariencia racional dentro del mundo sensible y lo que ésta presupone - la agencia-, devolviéndonos a un dualismo intraspasable que es también escisión racional de un yo que no puede apropiarse reflexivamente de sus creencias consigo mismo. Lo que está en juego es, por tanto, la integridad del agente cognitivo, la armonía entre nuestras creencias y las demandas de nuestra conciencia racional, armonía que si el juicio expresa, el escepticismo imposibilita. Si desde el punto de vista teórico el escéptico nos acusa de actuar irracionalmente, lo que la perspectiva práctica le 
responde es que es él quien piensa irracionalmente. Éste es el suelo existencial en el que se enraíza y adquiere sentido la cuestión escéptica.

De lo que se trata es de justificar racionalmente los goznes, pero con la condición de que dicha justificación no sea una prueba teórica de los mismos, algo que, en concordancia con el escepticismo, se considera imposible. Pienso que han sido Wright (2004, p. 50) y, de forma menos ambigua, Sosa (2011, pp. 154-7) quienes han elaborado el argumento más eficaz contra el escepticismo radical bajo restricciones lógicas tan estrictas que apenas dejan margen de maniobra. La presentación que sigue combina ambas perspectivas.

En primer lugar, debemos tener en cuenta que si no hay razones a favor de la existencia del mundo externo o de la fiabilidad regular de nuestras facultades, tampoco existen razones en contra de tales presuposiciones. Algo que el escéptico concede por el mero hecho de considerarlas fundamentales y, por supuesto, de recomendar en lo que a ellas se refiere la suspensión del juicio.

Se trata, por tanto, de considerar una situación original de conflicto y equilibrio de actitudes respecto a alguna proposición-gozne, situación de la que el escéptico forma parte. La pregunta es: ¿cuál es la actitud racional que debemos adoptar, por ejemplo, respecto a la existencia del mundo externo: afirmación, negación, o suspensión del juicio? Parecería que, dado que no se trata de una pregunta que - de acuerdo con todas las partes de la disputa - pueda decidirse teóricamente, es el escéptico quien rompe, a su favor, el equilibrio inicial. Sin embargo, esto presupone que la pregunta se ha formulado en el vacio, sin tomar en consideración criterios internos del ejercicio racional tales como la coherencia, no sólo en lo que se refiere a la relación que guarden entre sí las creencias de un sistema de primer orden (donde la incoherencia entre creencias es signo de irracionalidad), sino a la concordancia lógica transversal que debe existir entre nuestro sistema judicativo de primer orden y la actitud reflexiva que adoptemos respecto al mismo. Un sujeto que juzga en abstracto que su sistema concreto de juicios es másicamente falso no es coherente en la actitud que toma respecto a su pensamiento. Como tampoco lo es quien suspende reflexivamente el juicio — quien adopta una actitud escéptica de segundo orden - sobre su sistema de juicios. La conclusión es que, para salvaguardar la coherencia entre nuestros juicios y la evaluación general de los mismos, la única actitud racional que uno puede tomar respecto a las presuposiciones básicas implícitas en nuestra actividad judicativa es la afirmación. Las otras actitudes resultarían en una contradicción formal. 
Pese a la relevancia del argumento anterior, el escéptico cuenta con una buena respuesta. Podría decir, por ejemplo, que es precisamente porque prioriza el criterio de coherencia por lo que recomienda una suspensión general del juicio, que afecte tanto a los goznes y a la actitud general reflexiva como a toda actividad judicativa empírica. Y podría también añadir que dicha suspensión general no le impide actuar como un ser humano más, siguiendo los dictados de la naturaleza y la fuerza de las apariencias sin por ello comprometerse racionalmente con su verdad. Para el escéptico, la suspensión general armoniza el primer y el segundo orden, dotando de racionalidad interna a su posición.

Es, sin embargo, esta suspensión completa de la actividad racional (o lo que parece serlo) la puerta que se le abre al filósofo wittgensteiniano para entrar en el baluarte escéptico. El escéptico dice alcanzar sus conclusiones obedeciendo la norma epistémica fundamental: determina tu voluntad racionalmente. Sin embargo, uno puede determinarse a seguir dicha norma únicamente bajo el supuesto de que tal determinación sea efectiva en la práctica, es decir, bajo el supuesto de que no sólo sea posible querer racionalmente, sino actuar motivados por tal determinación. Abrigando dudas sobre los goznes, el escéptico cuestionaría la eficacia causal de la norma, y, por tanto, la posibilidad misma de la determinación racional de la voluntad. En otras palabras: es irracional querer lo que en la práctica es imposible; la racionalidad del escéptico se adquiere al precio del abandono del escepticismo; en el sentido señalado, los goznes son constitutivos de la propia racionalidad epistémica. Reaparece, por tanto, la conclusión del argumento anti-escéptico previo: la afirmación como única actitud racional posible respecto a nuestras creencias fundamentales.

Si consideramos brevemente el estatus epistémico que, de acuerdo con esta posición, poseen los goznes, podríamos decir, por una parte, y porque se trata de compromisos que son absolutamente ciertos y absolutamente necesarios (aunque con el inciso de que este 'absolutamente' no se extiende más allá de la racionalidad trascendental), que no se trata de simples opiniones arbitrarias, y, por otra, y porque su vínculo con la racionalidad es, aunque interno, puramente práctico, que se trata de postulados. De hecho, existe una similitud evidente entre los postulados (kantianos) de la razón práctica y —en el área de la racionalidad epistémica— los goznes. En ambos casos, el problema es el de tender un puente entre un imperativo incondicional (en un caso, moral; en el otro, epistémico) y la posibilidad de actuar de acuerdo con él, y, en consecuencia, lo que se plantea es la cuestión de la 
transición entre noúmeno y fenómeno. También en ambos casos, lo que se propone es una justificación a priori de la realidad de una serie de 'objetos' que, aunque teóricamente indemostrables, serían necesarios para la agencia en sentido estricto. El problema radica, no sólo en lo que las críticas a Kant puedan decirnos acerca de la validez de su justificación práctica de la realidad de objetos suprasensibles, sino, sobre todo, en las diferencias que, en detrimento de la epistemología de goznes, esta analogía general oculta. Por lo pronto, y como veremos en un momento, el escéptico en epistemología, y al contrario de lo que sucede en el caso del escéptico en moral, no pone en duda la eficacia de la voluntad racional y, por consiguiente, la existencia de actos libres — de determinación libre de nuestra actitud epistémicadentro del orden de la naturaleza.

Nótese, en primer lugar, que al tratarse de una justificación a priori el punto de partida del argumento es el hecho de que la norma epistémica 'determina racionalmente tu voluntad' es incondicional y posee validez a priori. En términos de Kant, es un hecho de la razón, y, como tal, se trata de un imperativo legítimo con independencia de cómo estemos empíricamente constituidos, de que las circunstancias sean propicias, o de que dicha determinación de la voluntad pueda o no implementarse en la práctica y producir un efecto real en el mundo sensible. Objetivamente, deber sólo implica deber. Lo que significa que, para deducir poder de deber, el argumento, lejos de ser puramente a priori, ha de contener, además de una premisa a priori, una premisa empírica - el hecho psicológico bruto de que nuestra constitución subjetiva es tal que somos incapaces de llegar a determinarnos racionalmente sin primero asumir la eficacia de la voluntad racional. Pero entonces se trata de un argumento relativo a condiciones empíricas (al perfil psicológico humano), lo que cancela la tesis de que los goznes son constitutivos de la agencia racional en sí misma y de que se trata de certezas universales, fundamentales y necesarias. Es importante destacar también que la universalidad de este hecho psicológico bruto es problemática, aunque solamente sea porque el conflicto entre la voluntad y su eficacia puede expresarse en diversas actitudes profundamente humanas, desde la ironía escéptica y la revuelta existencialista hasta la resignación trágica.

Sin embargo, existe un problema todavía mayor. Como ya dijimos, la función que para Kant cumplen los postulados de la razón práctica es la de vincular el imperativo categórico y la acción conforme al mismo. Sin embargo, el escéptico de goznes no solo no cuestiona que no podamos ejercer empíricamente la agencia 
racional, sino que actúa en todo momento de acuerdo con la norma epistémica, mostrando con su ejemplo que, pese a la presión que sobre nosotros ejercen las apariencias, siempre está en nuestro poder - $\mathrm{y}$, en consecuencia, que tenemos la libertad de - la suspensión del juicio. En otras palabras: la suspensión del juicio no es la suspensión de la actividad racional; por el contrario, se trata de la realización y satisfacción en concreto - dentro de la práctica empírica - de aquello a lo que la norma epistémica pura nos obliga. Por eso pienso que el ataque al escéptico se basa en un equívoco generalizado. No es que, de acuerdo con el escéptico, no estemos racionalmente obligados a comprometernos con la realidad de los goznes. Lo que estamos es racionalmente obligados a no comprometernos con ella. Y, por tanto, a una suspensión general del juicio que es el ejercicio de la libertad y de la determinación racional de la voluntad en el mundo sensible. Curiosamente, los postulados de la razón epistémica no sólo no vincularían norma y acción conforme a la norma, sino que, haciendo imposible la causalidad de la norma, instaurarían la misma escisión que supuestamente previenen: la división entre lo que el sujeto debe hacer — suspender el juicio- y lo que no puede llevar a cabo. Los goznes no son, por lo tanto, postulados de la razón, sino imperativos viscerales, o, mejor dicho, el objeto de nuestro corazón y de nuestros deseos. La constante determinación racional a la que se somete el escéptico ni siquiera concede el permiso (y mucho menos la obligación práctica) de creer en lo que no parece ser otra cosa que, como mucho, nuestros compromisos animales.

Como el problema tal como lo plantean los epistemólogos de goznes no es el del conflicto entre razón y sensibilidad, sino el de una paradoja interna a la racionalidad y el de una contradicción entre sus dimensiones teórica y práctica, y como la suspensión general del juicio logra reintegrar al sujeto epistémico con sus acciones, y, así, permite la concordancia entre ambas dimensiones de la razón, deberíamos pensar que el escéptico cumple lo que el dogmático sólo promete, y que, más que ofrecer paradojas, trata de aliviar las tensiones internas de la racionalidad. $\mathrm{Si}$, aun así, éstas se perpetúan, nada indica que se deban a un conflicto entre lo trascendental y lo empírico. Tal vez indiquen una disonancia constitutiva del propio yo y de su autonomía, de la unidad originaria (teórico-práctica) de la razón.

Lo que, en cualquier caso, hemos visto es que en toda situación de conflicto y equilibrio entre actitudes que incluya al escéptico entre sus partes, la equipolencia se resuelve automáticamente a favor de este último. No existe, por tanto, ni una justificación teórica ni una justificación puramente práctica (si es que esta última expresión tiene, después de lo que hemos desarrollado, algún sentido) de los goznes 
wittgensteinianos. Lo que significa que los argumentos expuestos no les otorgan legitimidad trascendental.

Con esta expresión me refiero, de acuerdo con el modelo kantiano de objetividad y necesidad, no a que no se haya demostrado que los compromisosgozne — en conformidad con el concepto pre-kantiano de objetividad- no estén dados a priori, sino a que no se ha justificado que sean o acciones necesarias de la mente o los productos necesarios de tales acciones. Lo que me lleva a preguntarme si, bloqueando esta vía, lo que el escéptico realmente nos muestra es que el único medio para la validación de la exterioridad es el de mostrar que ésta se encuentra incluida en la construcción del yo y la autoconciencia trascendental como su condición de posibilidad. La epistemología de goznes no cuenta, sin embargo, ni con las herramientas ni con la direccionalidad para afrontar un proyecto así. Un proyecto que no es otro que el de la Wissenschaftslehre.

O eso o el escepticismo.

\section{Quid Juris? Quid facti?}

Algo que se deduce del punto previo es que, considerado como una refutación del escepticismo, el argumento que Coliva propone - y que, recordemos, incluye entre sus premisas el hecho de que el escéptico admite la racionalidad de nuestras prácticas epistémicas- es invalido. El escéptico no acepta, como acabamos de ver, la legitimidad trascendental de los goznes, que se trate de productos a priori de la actividad racional, y, por ello mismo, que doten de legitimidad a priori a las prácticas que fundamentan y en las que se expresan. Aquí, no hay transmisión deductiva de justificación racional simplemente porque no hay justificación racional que pueda transmitirse.

Sin embargo, podría leerse la deducción trascendental de Coliva, más que como un argumento justificativo, como un argumento explicativo cuyo objeto fuese —con independencia de cuál sea el estatus epistémico de los goznes considerados en sí mismos o incluso de si poseen estatus epistémico- el de hacer explícita la relación de fundamentación y constitución que vincula a los compromisos-gozne con las prácticas epistémicas cotidianas, o, lo que es lo mismo, el de mostrar que los goznes son las reglas que describen la normatividad inherente al lenguaje y a la práctica epistémica, y que, de modo implícito, guian y determinan la formación de juicios. 
Los goznes serían, por tanto, ejemplos de lo que Wittgenstein llama proposiciones gramaticales (Schmitz, 2006, pp. 228-37). Como tales, ni conformarían un cálculo independiente de y previo a la práctica sobre el que ésta se construye ni se trataría de reglas que uno tuviese que aprender primero para así convertirse en usuario lingüístico o epistémico — como escribe Wittgenstein, "(e)1 lenguaje no ha surgido de proceso de razonamiento alguno" (Wittgenstein, 2004, $\S 475)$. Por el contrario, se trataría de reglas que sólo pueden ser comprendidas y empleadas dentro del horizonte vivo de la práctica, que expresan aspectos fundamentales de prácticas ya existentes, y que únicamente son formuladas en un contexto filosófico posterior a la práctica como descripciones o comentarios sobre la misma. Poner en cuestión esas reglas significa poner en cuestión la práctica en su totalidad: es precisamente a esto - a la relación interna y constitutiva de goznes y prácticas - a lo que el argumento de Coliva apunta.

Parecería, así, que el argumento está dirigido a una variedad muy específica de escepticismo, en la que el objeto de duda es la vinculación de regla y práctica (o que la práctica dependa de la regla), o, lo que es igual, en la que el problema que se plantea es, en una de sus acepciones, el de cual sea el criterio para distinguir seguir una regla y parecer que se sigue una regla. El constitutivismo de Coliva previene esta amenaza escéptica negando la distinción que la posibilita, entre reglas y prácticas. Las normas son, tal como insiste, inherentes a la práctica. Lo más importante, en cualquier caso, es que la cuestión ya no es la de la validez de la normatividad epistémica, sino la del papel que los goznes puedan desempeñar en la configuración de dicha normatividad, y, en consecuencia, la de si ésta se trata (y hasta qué punto se trata) de una normatividad objetiva o de una apariencia (contingente, subjetiva) de normatividad. Mientras la normatividad objetiva recogería principios $y$ hechos fundamentales que determinarían eventos en las carreras de los objetos, una normatividad subjetiva se limitaría a reflejar expectativas cuya base es la regularidad de la experiencia, tratándose de una apariencia de orden que no puede imponerse preformativamente sobre los fenómenos.

Las preguntas fundamentales son, por tanto, las de cómo y si las presuposiciones wittgensteinianas guían y determinan la formación empírica de juicios. Aunque me centraré en la segunda de estas cuestiones (Quid facti?) —referida a si los goznes cumplen una función determinadora en la asignación de conexiones epistémicas y categoriales bien entre los objetos de experiencia y los de juicio o entre fenómenos-, haré antes de nada una breve referencia a la primera (Quid Juris?). 
Quid Juris? El problema radica en que, de acuerdo con el modelo constitutivista, en las prácticas epistémicas se da por supuesta la síntesis de norma y sensibilidad, síntesis en la que los goznes desempeñan la función de principios de inteligibilidad que unifican y permiten interpretar en una dirección específica la multiplicidad y diversidad de los datos brutos de la experiencia sensible. La cuestión es la de cómo podemos aplicar a la experiencia normas que ni forman parte de, ni tienen su origen en los contenidos de la última, o, en otras palabras, el problema es el de cómo normas a priori, principios necesarios y universales análogos a las categorías del entendimiento, pueden construir objetos de conocimiento a partir de la materia prima que aparece como dada en la sensibilidad, y, por tanto, en cómo es posible la interacción entre los principios del entendimiento y los fenómenos de la sensibilidad.

No me cabe duda de que Coliva afrontaría este problema apelando al hecho de que, porque las normas son inherentes a la práctica y, en sus palabras, "no cuelgan en el aire, sino que dependen de nuestras prácticas" (Coliva, 2015, p. 129), principios y actividades no constituyen dos ámbitos independientes a la espera de que podamos tender un puente entre ellos. Nos enfrentaríamos, por tanto, a un pseudoproblema cuyo origen es la tesis intelectualista de que primero están las normas, y de que las prácticas se construyen algorítmicamente a partir de ellas.

Sin embargo, el problema no se refiere a la relación (constitutiva o constructiva) de normas y práctica, sino de normas y sensibilidad. Y aquí el constitutivista únicamente cuenta con dos opciones. Por una parte, podría decir que los compromisos-gozne son generalizaciones cuya base es la experiencia. Pero eso supondría que ni son certezas, ni principios universales y necesarios, ni elementos constitutivos de la agencia racional. De hecho, esta respuesta perdería la normatividad objetiva y la reemplazaría por una mera apariencia de orden, obligando al constitutivista a abandonar su posición. La alternativa es la confirmación de su estatus a priori. Pero entonces la pregunta acerca de cómo es posible la aplicación de lo a priori a la experiencia tiene sentido. De acuerdo con esta segunda opción, el constitutivismo de Coliva permanece firme, pero al precio de un dualismo epistemológico (y también, posiblemente, de un dualismo de facultades discretas) que parece la herencia inevitable del constitutivismo kantiano en el que se inspira.

Lo que, en cualquier caso, resulta evidente es que nos enfrentamos a una cuestión a la que no puede responderse apuntando al hecho de que, tal como la existencia de nuestras prácticas demuestra, la síntesis entre lo a priori y lo a 
posteriori es real. No se trata ahora de que el hecho mismo sea problemático. Aunque lo es, tal como veremos en un momento al discutir la cuestión Quid facti? Sino de que apelar a ese hecho es tan poco relevante para la solución del problema que hemos planteado como lo sería apuntar al hecho de que mente y cuerpo interaccionan para responder a la cuestión de cómo es posible - y de qué haría inteligible- esa interacción. Lo que sucede es que la comprensión e inteligibilidad del 'cómo' de la síntesis nos están vedadas; que en lo que respecta a los objetos empíricos no disponemos ni del fundamento requerido para la aplicación de las certezas wittgensteinianas ni de percepción de la relación de determinación en la que esta aplicación consistiría. Lo que es opaco para el entendimiento es, por tanto, la propia constitución de la inteligibilidad de la experiencia, o, lo que es igual, la constitución e inteligibilidad de las prácticas epistémicas.

El problema más importante es, sin embargo, diferente. ¿Es verdad, tal como insisten los wittgensteinianos, que goznes y prácticas se encuentran tan íntimamente unidos que la duda sobre los primeros implica automáticamente poner en cuestión (y dejar sin explicación) las segundas? ¿Es cierto que Wittgenstein ha demostrado que, para que las prácticas epistémicas sean posibles, es necesario, no sólo que apliquemos, sino que seamos capaces de aplicar, la creencia de que existe un mundo material externo o el concepto general de causalidad a fenómenos concretos dados? Quid facti?

Es imprescindible, en primer lugar, que clarifiquemos los términos para el planteamiento del problema, entre otras cosas, para así distanciarnos de un escepticismo (hipotéticamente) humeano y para que lo que propongamos sea verdaderamente una crítica inmanente de la epistemología wittgensteiniana de goznes.

Por lo pronto, las nociones que emplearemos de 'fenómeno' y de 'experiencia' no se corresponden con la concepción mínima de las mismas habitual en el empirismo, donde la experiencia es una mera fantasmagoría o flujo de imágenes mentales cuyo contenido (si es que lo poseen) es puramente sensorial o fenoménico y donde los únicos datos que el escéptico parece aceptar se corresponden con las 'sensaciones' kantianas. Una concepción así de la experiencia es inconcebible, en el sentido de que se trataría de una experiencia tal que sus 'objetos' - al no encontrarse formalmente determinados — no serían susceptibles de aprehensión, y, por ello, no serían nada para nosotros. La conciencia empírica, para ser tal, requiere intuiciones 
a las que podamos diferenciar en virtud de relaciones espacio-temporales y ubicar dentro de un encuadre objetivo sin el que no sería posible ni su presentación en la conciencia ni determinaciones (o predicaciones) tan simples de una sensación como 'aquí' o 'ahora'. La experiencia, tal como la contempla el empirista, es vacía. Lo que indica que, para que la particularidad de la sensación no se hunda en el abismo de lo inefable, se requiere una red de términos universales. Por eso, entenderemos 'experiencia' en un sentido denso y formalizado, y 'fenómeno' con un significado análogo (aunque no necesariamente idéntico) al que Kant le dio a esta noción. No reflexionaremos sobre la función de los goznes respecto a una experiencia indeterminada (e inexistente), sino sobre la relación de goznes e intuiciones que ya se encuentran formalmente constituidas (tal como se presentan - secuencial y espacialmente- en la conciencia ordinaria).

Esto significa que, en concordancia con Wittgenstein, el escéptico al que consideraremos acepta que, como poco, los goznes son condiciones necesarias de la experiencia, y que, por tanto, se trata de normas o reglas que establecen la posibilidad de un objeto de experiencia (y de pensamiento) en general. La cuestión es la de si, además de establecer la posibilidad general de ese objeto, también se trata de reglas en virtud de las cuales se determinan objetos particulares de experiencia. ¿Los goznes se limitan a ser formas trascendentales de pensamiento, o, por el contrario, son principios activos de la práctica epistémica, que constituyen y regulan evidencias? ¿Constituyen la práctica porque hacen posible la experiencia, o la práctica tiene cuidado de sí, independientemente de cuáles sean las condiciones de la experiencia en general?

Prestemos atención, en primer lugar, al principio general de causalidad —una de las presuposiciones semánticamente sustantivas que Wittgenstein menciona explícitamente. Lo que ese principio nos dice es que la experiencia, para ser tal, debe adecuarse a leyes causales, de modo que es necesario que exista una razón suficiente para todo evento. Lo que, sin embargo, no nos dice es qué eventos son causas, siendo como principio compatible tanto con el que el humo sea causa del fuego como con que el fuego sea causa del humo. Lo que significa que si deseamos saber qué eventos ejemplifican la relación causal y cómo lo hacen, tenemos que recurrir a la experiencia.

El problema es que, como el propio Wittgenstein insiste en coincidencia con Hume (Wittgenstein, 2004, $\S ~ 130,133-35,240,275)$, la experiencia no nos sirve 
de ayuda alguna. Lo que en el mejor de los casos ésta muestra es una conjunción constante de fenómenos, pero no una conexión universal y necesaria entre los mismos. Lo que implica, para cualquier caso concreto que consideremos, que nunca podemos estar seguros de que realmente se trate de una ejemplificación de y de una aplicación a la experiencia de la relación causal — por no decir que, limitadas al pasado (Wittgenstein, 2004, $\S 275$ ), tales conjunciones ni pueden proyectarse como necesidades hacia el futuro ni, en consecuencia, pueden expresarse más que como hipótesis de cuya necesaria ausencia de prueba se sigue "que siempre nos reservamos el derecho a desecharlas o alterarlas" (Waismann, 1979, p. 100). La consecuencia es que incluso aunque el humo siempre siga al fuego o aunque el agua nunca se haya congelado al fuego (Wittgenstein, 2004, § 613), no podemos inferir ni que el fuego sea causa del humo ni que, por mucho en que insistamos en que debe existir una explicación desconocida para tal evento en caso de producirse, el agua no pueda congelarse al fuego. Por lo tanto, y aunque sea verdad que la experiencia no es posible sin asumir el principio general de causalidad, éste es tan indeterminado que no podemos saber para ningún caso específico si se aplica. Y si no sabemos dónde y cuándo se aplica también desconocemos si realmente lo hace.

Lo importante, sin embargo, es que estas consideraciones escépticas no afectan en nada a la práctica y al uso diario de terminología causal, contexto (el ordinario) en el que nos conformamos con hipótesis y conjunciones constantes allí donde nunca podemos establecer relaciones necesarias. Con lo que contamos es, por tanto, con una explicación deflacionaria de nuestro lenguaje causal compatible con el escepticismo acerca de la realidad objetiva (del uso o del significado) del principio de causalidad — de que realmente se trate de una regla de determinación de la experiencia. Contrariamente a lo que señala la deducción trascendental de Coliva, podemos dudar de que la regla constituya la práctica y guie nuestros juicios causales sin que la práctica desaparezca por eso. La práctica no se sostiene sobre fundamentos estables.

Algo similar ocurre con la creencia básica en la realidad del mundo material externo. Lo que este principio nos dice es que la posibilidad general de que la experiencia posea peso y direccionalidad epistémicas depende de dicha presuposición, o, lo que es igual, que es necesario que exista un mundo externo para que alguna experiencia pueda contar como razón de una creencia. Podríamos, incluso, extender la interpretación del principio, señalando que lo que éste afirma es que es necesario que nuestras creencias sean masivamente verdaderas para que haya experiencias que cuenten como evidencias. Lo que, sin embargo, no nos 
dice es ni qué experiencias cuentan como razones o indicadores objetivos ni, en caso de hacerlo, qué es lo que indicarían (como si la mera existencia del mundo externo pudiese prevenir una irregularidad futura en la naturaleza que cancelase el peso epistémico en una dirección específica de la experiencia pasada). En otras palabras: la presuposición del mundo externo es irrelevante tanto para determinar si mi experiencia actual es una percepción o una ilusión como para servirnos de guía en el supuesto de que un fenómeno inesperado contradijese nuestra experiencia pasada y nos viésemos obligados a reconsiderarla (Wittgenstein, 2004, § 503).

De nuevo, parecería como si para formar juicios concretos tuviésemos que consultar la experiencia. Y de nuevo, el problema radica, como también nos recuerda en numerosas ocasiones Wittgenstein $(2004, \S \S 362,368)$, en que la experiencia es incapaz por sí misma de determinar la relación entre objeto de experiencia y objeto de creencia y de regular el peso respectivo de evidencias contradictorias, y en que su carácter indeterminado o abierto nos obliga, en caso de conflicto, a tomar decisiones en el vacío. Si un árbol se transformase súbitamente en un hombre (Wittgenstein, 2004, § 513) podríamos pensar que se trata de una ilusión, o, tal vez, que lo que era ilusoria era la percepción del árbol, o, tal vez, y dado que ni el principio de causalidad general ni la creencia indeterminada en la realidad del mundo externo prescriben nada acerca de lo que sea o no posible en la naturaleza, que tales alteraciones podrían ocurrir, y, por tanto, que ambas impresiones - la pasada y la presente - son verídicas (y no hay conflicto entre ellas).

Lo importante es que la experiencia deja indeterminado ad infinitum el valor de la creencia, de forma que ésta última no pasa de ser más que una mera hipótesis, y, además, una hipótesis que jamás puede ser definitivamente confirmada. Y que, pese a que la presuposición del mundo externo no cumple función alguna en la formación de juicios, y a que estos son arbitrarios, las prácticas judicativas ordinarias no se resienten. La regularidad limitada y contingente de la experiencia proporcionaría una explicación escéptica de nuestro lenguaje justificativo.

Baste señalar finalmente que el mismo esquema argumentativo se repite respecto a la presuposición de un orden temporal objetivo —otro de los goznes wittgensteinianos a los que nos hemos referido. Aunque este principio dice que necesariamente todo evento ha de ser ubicado en una secuencia temporal, lo que no determina es qué posición debe ocupar un evento dado en esa secuencia. Algo que tampoco determina la experiencia. De hecho, tanto el principio como los 
datos empíricos de los que disponemos son compatibles con la posibilidad de que la experiencia actual sea la primera de la serie (la llamada 'hipótesis de Russell', a la que Wittgenstein hace referencia en 2004, $\S \S 183-95)$, y, por tanto, con la posibilidad de la ilusión del pasado. Lo que remite, también en este caso, y pese al marco o regla que establece la posibilidad general de objetos y juicios temporales, a la constitución subjetiva de nuestros juicios temporales empíricos.

En los tres casos que hemos analizado partimos del carácter formal y vacío de los compromisos-gozne, y nos preguntamos sobre los materiales de los que echamos mano para construir nuestras actividades judicativas cotidianas. Estos materiales acaban siendo arbitrarios y subjetivos. Sin embargo, que esto explique escépticamente cómo actuamos no significa que lo que hemos proporcionado sea una respuesta escéptica al escepticismo. Que la práctica pueda explicarse deflacionariamente no implica ni que sea inteligible ni que sea fuente de inteligibilidad.

El problema es que si, por una parte, los goznes instituyen un pensamiento puramente formal en el que nos limitamos a pensar un objeto material simplemente a través de formas lógicas y abstractas de cognición (por lo que no hay objeto concreto de pensamiento), por otra, el objeto concreto de pensamiento que construimos a partir de la experiencia es una mera combinación accidental (sin síntesis) de elementos independientes, y el pensamiento que a él se refiere es, así, puramente arbitrario — de modo que, porque no comprendemos el objeto que decimos pensar, realmente con él ni pensamos ni entendemos nada. En resumen: el así llamado 'objeto de experiencia' no es una unidad ni para la sensibilidad ni para el entendimiento. Y, por supuesto, únicamente a partir de principios gramaticales $\mathrm{y}$ formales no puede construirse unidad concreta alguna.

Fluctuando entre un pensamiento vacío y el no-pensamiento, de lo que carecemos es de pensamiento real o sintético, de reglas a partir de las que pueda construirse, no un objeto de experiencia en general, sino objetos determinados inteligibles. Quid facti? ¿Qué síntesis? ¿Qué pensamiento? Mientras que la normatividad a priori a la que apela Coliva no genera objetos de conocimiento, la experiencia que explica nuestras prácticas empíricas no proporciona objetos de pensamiento. Igual que del apriorismo kantiano, del apriorismo wittgensteiniano únicamente se sigue el escepticismo empírico. 


\section{Conclusión}

Durante las reflexiones anteriores he hecho referencia a algunos de entre los numerosísimos textos que demuestran que Wittgenstein fue claramente consciente del problema que hemos analizado. Uno de los pocos intérpretes que ha concedido a esos textos la relevancia que poseen, John Cook (1994) piensa que la función de la teoría wittgensteiniana de los goznes es, precisamente, la de prevenir las conclusiones escépticas que se deducirían de la contingencia, arbitrariedad e irregularidad posible de la experiencia (Cook, 1994, pp. 221-24). Piensa también, por supuesto, que, porque los goznes son incapaces de garantizar una normatividad objetiva empíricamente relevante, el proyecto de Wittgenstein fracasa.

Creo, sin embargo, que la lectura de Cook - inspirada en una concepción fenomenista de Wittgenstein (Cook, 1985, pp. 112-13) que, además de incorrecta, desatiende las raíces kantianas de su pensamiento, y haciendo caso omiso al hecho (entre otros) de que Wittgenstein ni apela a los goznes para responder a las hipótesis escépticas empíricas que desarrolla ni tan siquiera parece proporcionar respuesta alguna a las mismas - revierte al completo el orden lógico de Sobre la certeza. Es la propia teoría de goznes la que, en su interior, y al igual que había ocurrido con el trascendentalismo kantiano, abre espacio a una variedad radical de escepticismo empírico. Por eso, lo que Wittgenstein descubre es que, pese a las potencialidades anti-escépticas de su teoría tanto en lo que se refiere a un escepticismo trascendente basado en la posibilidad de la cosa-en-sí como a un escepticismo que afecta al carácter último de la experiencia y de sus condiciones de posibilidad, ésta (su teoría de goznes) se encuentra lógicamente vinculada a un tipo de escepticismo que, aunque generalmente desapercibido, tiene consecuencias devastadoras. Si este escepticismo es (o no) asumible, si se trata de una posición con la que debemos aprender a convivir, o, por el contrario, de una opción que deba ser refutada y que sólo pueda serlo mediante un cambio radical de perspectiva filosófica, son cuestiones que Wittgenstein no pudo llegar a elaborar. Como poco, la hermenéutica wittgensteiniana debería, más que ocultarlas, hacerlas nuevamente visibles.

Lo que hemos constatado, en fin, es la dualidad entre principios a priori que al no determinar la experiencia nos devuelven un pensamiento vacio, y prácticas cuyo fundamento es la constitución arbitraria de objetos, constitución que lo 
único que nos proporciona es una materia cognitiva que no podemos pensar. En un sentido, este escepticismo semántico extremo nos retrotrae al problema cartesiano de las ideas materialmente falsas y de la opacidad intelectual de las sensaciones. En otro, nos obliga a reconsiderar las condiciones del pensamiento real concreto, es decir, de los principios de juicio como principios de posibilidad de la predicación sintética. Pero ése es precisamente el tema del idealismo especulativo de Fichte y Hegel.

Las deficiencias kantianas de Wittgenstein que el escepticismo trae a la luz podrían ser también la ocasión para que reconsideráramos la opción idealista.

\section{Referencias}

Barnes, J. (1990). The Toils of Scepticism. Cambridge: Cambridge University Press.

Coliva, A. (2015). Extended Rationality: A Hinge Epistemology. Basingstoke: Palgrave.

Cook, J. W. (1985). The Metaphysics of Wittgenstein's On Certainty. Philosophical Investigations, 8(2), 81-119.

Cook, J. W. (1994). Wittgenstein's Metaphysics. Cambridge: Cambridge University Press.

Fichte, J. G. (1988). Early Philosophical Writings (D. Breazeale, Trans.). Ithaca \& London: Cornell University Press.

Plato. (2014). Theaetetus. Oxford: Oxford University Press.

Pritchard, D. (2016). Epistemic Angst: Radical Scepticism and the Groundlessness of Our Believing. Princeton \& Oxford: Princeton University Press.

Schmitz, B. (2006). Grammatical Propositions. En M. Kober (Ed.), Deepening Our Understanding of Wittgenstein (pp. 227-49). Amsterdam \& New York: Rodopi.

Sosa, E. (2011). Knowing Full Well. Princeton and Oxford: Princeton University Press.

Sosa, E. (2015). Judgment and Agency. Oxford: Oxford University Press.

Waismann, F. (1979). Wittgenstein and the Vienna Circle. Oxford: Blackwell.

Wittgenstein, L. (2004). On Certainty. Oxford: Blackwell.

Wright, C. (1991). Scepticism and Dreaming: Imploding the Demon. Mind, 100(397), 87-116.

Wright, C. (2004). Wittgensteinian Certainties. En Dennis McManus (Ed.), Wittgenstein and Scepticism (pp. 22-55). London \& New York: Routledge.

Wright, C. (2012). Replies Part IV: Warrant Transmission and Entitlement. En Annalisa Coliva (ed.), Mind, Meaning, and Knowledge: Themes from the Philosophy of Crispin Wright (pp. 451-86). Oxford: Oxford University Press. 